review 107 papers were included to examine serum biomarkers to predict short term outcome.

Raised mean serum levels of IL-6 (p value 0.04, 95\% CI 44.5 to -0.66 ) and lactate ( $\mathrm{p}$ value $<0.001,95 \%$ CI -6.19 to -2.81) are associated with worse short term outcome in NE. The large confidence intervals, however, reflect the small number of studies included and consequently the small population cohorts. Multiple other meta-analyses had substantial heterogeneity and were not statistically significant. A large proportion of studies were excluded due to variety of measured outcomes and methods of data reporting. Establishment of Core Outcomes in NE will benefit future research.

\section{OC62 THE IMPACT OF CHORIONICITY AND ASSISTED REPRODUCTIVE THERAPIES IN OBSTETRIC AND NEONATAL OUTCOMES: A RETROSPECTIVE STUDY IN 1783 PORTUGUESE TWINS}

${ }^{1}$ Catarina Ferraz Liz*, ${ }^{2}$ Sara Domingues, ${ }^{2}$ Ana Guedes, ${ }^{2}$ Luísa Lopes. ${ }^{1}$ Centro Hospitalar Tâmega e Sousa, Penafiel, Portugal; ${ }^{2}$ Centro Materno Infantil do Norte, Porto, Portugal

\subsection{6/archdischild-2019-epa.59}

Introduction Multiple gestations' incidence has risen worldwide in the last years, much due to assisted reproductive therapies (ART).

Monozygotic twins have been associated to adverse neonatal outcomes, however the effect of ART is still unknown.

The goal of this study was to analyze obstetric and neonatal outcomes of twin pregnancies in a level 3 maternity.

Methods A retrospective study including all twins born in a period of 12 years (2003-2014) in a level 3 maternity was conducted. Data were collected from clinical records.

Analysis comparing spontaneous monochorionic and dichorionic twins and spontaneous and ART dichorionic twins were performed.

Results The sample included 1783 newborns from 875 mothers. Mean maternal age was 31 years, with 616 spontaneous pregnancies and 259 through ART.

Prematurity occurred in $77 \%$. Congenital malformations were found in $6 \%$, with cardiac malformation being the most common $(n=48)$, followed by reno pelvic $(n=40)$ and skeletal $(n=29)$. Mortality rate was 3\% $(n=51)$.

- Spontaneous monochorionic and dichorionic twins

A total of 1202 newborns were found, 428 monochorionic and 774 dichorionic.

Gestational diabetes was more common in dichorionic pregnancies (9\% vs $5 \%, \mathrm{p}=0.009)$.

Monochorionic twins had higher prematurity $(79 \%$ vs $72 \%, p=0.017)$ and very low birthweight (VLBW) rate $(19 \%$ vs $14 \%, \mathrm{p}=0.015)$.

Congenital anomalies ( $9 \%$ vs $6 \%, \mathrm{p}=0.022)$, especially cardiac malformations ( $4 \%$ vs $2 \%, \mathrm{p}=0.013)$, Respiratory Distress Syndrome $(23 \%$ vs $18 \%, \mathrm{p}=0.049)$, patent ductus arteriosus $(7 \%$ vs $4 \%, \mathrm{p}=0.021)$, anemia $(11 \%$ vs $5 \%$, $\mathrm{p}<0.001)$, periventricular hemorrhage ( $5 \%$ vs $3 \%, \mathrm{p}=0.047)$, cardiac $(7 \%$ vs $4 \%, p=0.019)$ and surgical $(4 \%$ vs $2 \%$, $\mathrm{p}=0.026)$ diseases, mechanical ventilation $(16 \%$ vs $10 \%$, $\mathrm{p}=0.001)$ and mortality $(4 \%$ vs $2 \%, \mathrm{p}=0,015)$ were higher in monochorionic twins.

- Dichorionic spontaneous and ART twins

The sample included 1264 newborns (770 spontaneous and 464 ART).
Maternal age $>35$ years $(35 \%$ vs $27 \%, \mathrm{p}=0.006)$, gestational diabetes $(14 \%$ vs $9 \%, \mathrm{p}=0.006)$ and hypertension $(12 \%$ vs $7 \%, \mathrm{p}=0.002$ ) were more common with ART.

Alcohol (1 vs 0\%, p=0.028) and tobacco $(18 \%$ vs $6 \%$, $\mathrm{p}<0,001)$ consumptions were higher in spontaneous pregnancies.

Prematurity $(72 \%$ vs $79 \%, \mathrm{p}=0.01)$ and VLBW $(18 \%$ vs $14 \%, \mathrm{p}=0.03$ ) were higher in ART twins.

There was no difference in the rate of congenital malformations.

Conclusion As described in the literature, monochorionic pregnancies were associated to worst obstetric and neonatal outcomes.

In this study ART was associated with higher risk of prematurity and very low birthweight. Unlike what has been described, ART was not associated with congenital anomalies.

\section{OC63 NEONATAL ABSTINENCE SYNDROME (NAS): HOW THE TIMING OF PRESENTATION INFLUENCES A SAFE DISCHARGE POLICY}

${ }^{1,2}$ Mugahid Ibrahim*, ${ }^{2}$ Rajesh Pandey, ${ }^{2}$ Sydney Stark, ${ }^{2}$ Ahmad Ali, ${ }^{2}$ Kelly Gibson,
${ }^{2}$ Jennifer Bailit, ${ }^{2}$ Aparna Roy, ${ }^{2}$ Deepak Kumar, ${ }^{1,3}$ Roy K Philip. ${ }^{1}$ University Maternity Hospital
Limerick, Limerick, Ireland; ${ }^{2}$ MetroHealth Medical Center/Case Western Reserve University,
Cleveland, Cleveland, USA; ${ }^{3}$ Graduate Entry Medical School, University of Limerick, Limerick,
Ireland

10.1136/archdischild-2019-epa.60

Background Incidence of NAS from in-utero exposure to opioids has increased dramatically over the years in parallel with the general opioid uptake in societies. Management of NAS places social, emotional and financial burden on families and the health care systems. Age at which symptomatic NAS require pharmacological treatment is reported variably. Consequently, there is lack of consensus regarding the duration of observation to ensure safe discharge of the infant.

Objective To determine the optimal duration of postnatal hospital observation for at- risk infants following in-utero exposure to opioids.

Design/Methods A retrospective 5 year review of infants $(\geq 36$ weeks gestation) born between 2013-2017 to mothers with opioid dependency. Maternal and infant characteristics were abstracted from patient records and electronic database of the Mother and Child Dependency Program at Metro Health System, Cleveland, Ohio, USA. Age in hours of life (HOL) when pharmacological treatment was started for NAS as per guidelines was noted and confidence interval was defined. We compared infants who required pharmacological treatment within 96 to those who were treated beyond 96 hours using nonparametric tests.

Results Of the 355 infants with in-utero opiate exposure studied, 255(88.4\%) required pharmacological treatment for NAS. Medications were initiated at a median age of 44.5 HOL (IQR 23-74.5 hours). 90 $\pm 3 \%$ of infants were commenced on pharmacological treatment by $96 \mathrm{HOL} ; 93 \pm 3 \%$ by $120 \mathrm{HOL}$ and $98 \pm 1 \%$ by $168 \mathrm{HOL}$ (Figure-1). Infants who received treatment within $96 \mathrm{HOL}(\mathrm{n}=199)$ compared to those treated at $>96 \mathrm{HOL}(n=26)$ were more likely to have a urine toxicology positive for both mother and baby, have higher mean NAS score in first week of life, require a higher maximum dose of morphine and longer hospital stay. After adjusting for gestational age, breast milk exposure, methadone vs. buprenorphine maintenance programme, NICU vs. Special Care Unit admission; infants and mothers negative for drug 
screen (from those with history of in-utero exposure) were significant predictors for onset of NAS requiring pharmacological intervention at $>96 \mathrm{HOL}$ (OR 0.21 ; p value 0.011 ).

Conclusions The majority of infants who required pharmacological treatment for NAS during their postnatal observation period were diagnosed within the first $120 \mathrm{HOL}$. Those atrisk infants, born to mothers with a known history of exposure, who have a negative urine toxicology screen for both baby and mother, should be monitored beyond 5 days as they tend to have a later presentation.

\section{OC64 STETHAID: MOBILE TECHNOLOGY FOR SMART AUSCULTATION IN CHILDREN}

'Layla Taryam, ${ }^{1}$ Sumaya AL Zarooni, ${ }^{1}$ Olfat Alzaabi, ${ }^{1}$ Asif Afridi, 'Zainab Abdullah, ${ }^{1}$ Amrish Kumar Kumar, ${ }^{1}$ Mohamed Ali Ali, 'Moza Khalaf, ${ }^{2}$ Titus John, ${ }^{2}$ Ravi T Ambati, ${ }^{2}$ Robin W Doroshow, ${ }^{2}$ Raj Shekhar, ${ }^{1}$ Asma Alzumar*. 'Ministry of Health and Prevention, Dubai, UAE; ${ }^{2}$ Children's National Health System, Washinton, DC, USA

10.1136/archdischild-2019-epa.61

Innocent heart murmurs occur in approximately half of healthy children at some point in their childhood. Still's murmur is the most prevalent of these innocent pediatric heart murmurs with a prevalence of approximately $40 \%$. Auscultation remain the primary clinical tool utilized to identify Still's murmur from the murmurs of true heart disease. However, general physicians (GPs) are not successful in distinguishing a Still's murmur from pathological murmurs and therefore refer a large number of children to pediatric cardiologists. Besides cost and inconvenience, these referrals are a source of extreme anxiety and stress in children and families while waiting to see a pediatric cardiologist. Our objective has been to develop a novel technology that can help a GP classify a Still's murmur with high accuracy at the point of care and support their decisions regarding referral to a specialist. We have created a stethoscope that connects to a smartphone and developed a custom mobile application that records heart sounds and, using machine learning, analyzes them for the presence of a Still's murmur. This solution is called StethAid. The algorithm to identify Still's murmur has been developed and tested utilizing a pediatric heart murmur library of over 1800 patients with clinically documented diagnoses compiled at Children's National Health System. Using StethAid, we recorded heart sounds of 312 pediatric patients at two UAE Ministry of Health and Prevention hospitals to be utilized as an independent test set. The algorithm identifies Still's murmur with a sensitivity of $89 \%$ and specificity of $97 \%$. The proposed technology could potentially lower the current high rate of referrals to pediatric cardiologists associated with Still's murmur.

\section{OC65 NORMATIVE NEONATAL FACIAL BIO-METRICS IN THE UNITED ARAB EMIRATES FOR NON-INVASIVE EARLY DETECTION OF GENETIC DISORDER}

${ }^{1}$ Olfat Al Zaabi*, ${ }^{2}$ Antonio R Porras, 'Safyia Saif Al Khajeh, 'Yamen El Moghanni, ${ }^{1}$ Mona Salah, ${ }^{1}$ Ahmed Abdelwahab Ismail, ${ }^{1}$ Asma Abdelrahman Al Mazar, ${ }^{1}$ Mona Abdul Rahman, ${ }^{1}$ Rola Helmi, 'Luay A Wahab, ${ }^{1}$ Hussein Eleimy, ${ }^{1}$ Anshad Ummerkhan, ${ }^{2}$ Marius George Linguraru. 'Ministry of Health and Prevention, Dubai, UAE; ${ }^{2}$ Children's National Health System, Washington, DC, USA

10.1136/archdischild-2019-epa.62
Perinatal identification of genetic syndromes with facial dysmorphology is challenging due to subtle manifestations and population variability. Recent studies have shown that genetic syndromes manifest differently on populations of different ancestries. The purpose of this study is to quantify the distribution of facial biometrics of healthy newborns among the local population of the United Arab Emirates. In this first study of its kind, we collected frontal facial pictures from 504 healthy newborns without facial dysmorphology (252 males and 252 females, age range 0-4 days, gestational age at birth range 37-42 weeks) between October 2015 and March 2017 at hospitals in the network of the United Arab Emirates Ministry of Health and Prevention: Al Qassimi Hospital in Sharjah and Fujairah Hospital. We measured a set of distances and angles between facial landmarks on the eyes, nose and mouth in all pictures. Horizontal and vertical distances were normalized as a percentage with respect to the distance between lateral canthi and the eye-to-mouth distance, respectively. The average axes of the palpebral fissures were $27 \pm 3 \%$ and $9 \pm 3 \%$. The distance between the medial canthi was $47 \pm 4 \%$. The average angle between each medial canthus with respect to the corresponding lateral cathus and the other medial canthus - a measure of slanting of the eyes-was $176 \pm 4$ degrees. The distance between the oral comissures was $48 \pm 6 \%$. The nose length was $35 \pm 8 \%$. This first normative reference of facial biometrics in newborns in the United Arab Emirates has great potential to support the perinatal identification of genetic conditions through quantitative facial analysis.

\section{OC66 SUCCESSFUL PILOT INTRODUCTION OF A 'VIRTUAL CLINIC' FOR A REGIONAL COHORT OF PATIENTS WITH TYPE 1 DIABETES}

${ }^{1}$ Anne Quinn, ${ }^{1}$ Therese Dunne, ${ }^{1,2}$ Clodagh O'Gorman, ${ }^{2}$ Niall Collins, ${ }^{1}$ Orla Neylon*. ${ }^{1}$ University Hospital Limerick, Limerick, Ireland; ${ }^{2}$ GEMS Medical School, UL, Limerick, Ireland

\subsection{6/archdischild-2019-epa.63}

Background Among Irish paediatric centres, 30\% are not meeting the recommendation of three-monthly review of patients with Type 1 Diabetes (T1D). ${ }^{1}$ Long waiting times are encountered by our patient cohort at out-patient clinics, along with a sometimes passive approach to reflective dose adjustment. We wished to explore the feasibility of a targeted educational intervention using a 'virtual clinic' in a sample of our population, given the well-described challenges with rural broadband provision in the West of Ireland.

Aim Primary outcome was the proportion of successfully completed internet 'virtual clinic' appointments with participants, including successful pre-clinic download of insulin pump data. Secondary outcomes included setting adjustments, impact on glycaemic control, appointment duration and user satisfaction, the latter by qualitative survey.

Methods Participants using insulin pumps and continuous glucose monitoring systems were recruited from out-patients. Intervention consisted of 3 'virtual clinic' sessions at 10-14 day intervals using 'Skype for Business@)' to connect the diabetes team with the family in their home. Data were collated in Excel, with postal HbA1c. 